\title{
VALIDACIÓN EMPÍRICA RESIDUAL CON USO DE SIMULACIÓN PARA EVALUAR CONVERGENCIA DE ALGORITMOS DE OPTIMIZACIÓN EN CALL CENTERS
}

\author{
Ángel Rubén Barberis ${ }^{1}$ y Lorena E. Del Moral Sachetti ${ }^{2}$ \\ ${ }^{1}$ Universidad Nacional de Salta, Sede Central - Salta Capital - Argentina \\ ${ }^{2}$ Universidad Nacional de Salta, Sede Regional Orán - Salta - Argentina
}

\begin{abstract}
RESUMEN
La optimización de Call Centers no es un problema fácil de resolver, debido a la complejidad de los modelos matemáticos que derivan de las fórmulas de Erlang. Esta complejidad se traslada a modelos de optimización, que en la mayoría de los casos, se conforman con funciones objetivos no lineales y no derivables. Así como en todas las áreas de la Investigación de Operaciones, resolver estos problemas demanda algoritmos eficientes, rápidos y precisos. La Simulación como herramienta experimental constituye un ambiente esencial para la validación de algoritmos de optimización, sobre todo cuando no se dispone de repositorios de problemas bien definidos con métricas de resultados conocidos con los que se pueda contrastar. En este trabajo se presenta una estrategia denominada validación empírica residual mediante el uso de simulación para dar credibilidad estadística de la convergencia de algoritmos de optimización no lineal entera en el estudio de los problemas de Call Centers.
\end{abstract}

PALABRAS CLAVE

Optimización de Call Center, Optimización no Lineal Entera, Convergencia de Algoritmos no Lineales

\section{INTRODUCCIÓN}

La optimización de recursos operativos en los problemas de Call Centers constituye un proceso difícil de resolver con precisión, debido principalmente a la complejidad de los modelos de Erlang, sobre todo cuando se intenta aproximar éstos a la realidad. Si bien, las formulaciones matemáticas para los modelos de Colas Erlang-A $(\mathrm{M} / \mathrm{M} / n+\mathrm{G})$ están disponibles, éstas son demasiadas complicadas para derivar de ellas soluciones analíticas y algorítmicas para problemas de Call Centers. Por lo que, resolver cuestiones como la estimación de la cantidad adicional de operarios cuando el volumen de llamadas entrantes se duplican, o la determinación de la sensibilidad del modelo cuando subyacen errores en la estimación de la paciencia (Zeltyn et al., 2005), entre otros, suele ser una tarea difícil.

La administración óptima de Call Center persigue, principalmente, dos objetivos contrapuestos: 1) Dimensionamiento con el menor costo en la contratación de personal (menor cantidad de personal, menor nivel de servicio); y 2) Maximizar los niveles de servicio, que se traduce en la maximización de la satisfacción del usuario (mayor nivel de servicio, implica mayor número de personal a contratar). Para el primer objetivo, se resuelven problemas de optimización lineal entera. En la literatura hay un amplio desarrollo de investigaciones y algoritmos eficientes sobre la temática (Atlason et al., 2007, Caprara et al., 2003, Ingolfsson et al., 2010, Robbins et al., 2010). Alcanzar el segundo objetivo, implica resolver un problema de optimización no lineal entero, para el cual, no existe un algoritmo general que pueda aplicarse en la búsqueda de la solución, por lo que se recurre al desarrollo de algoritmos específicos. En la literatura, se registran trabajos en el que se realiza un abordaje conjunto, priorizando la solución al primer objetivo, y en segundo lugar, realizan una aproximación de la solución al segundo objetivo (Ingolfsson et al., 2002, Koole et al., 2003). Los inconvenientes matemáticos dificultan el desarrollo de procesos analíticos generales que puedan demostrar convergencia de algoritmos de optimización no lineales como consecuencia de la diversidad de enfoques con que se abordan la problemática. La dificultad es aún mayor al no contar, en esta 
área de investigación, con repositorios de problemas bien definidos, con métricas de resultados conocidos con los que se pueda contrastar. En tanto, otros utilizan la simulación como una herramienta de comparación, apoyados con intervalos de confianza (Kim et al., 2011).

En los problemas de Call Centers, es muy común el uso la simulación, dado que es una excelente herramienta de soporte en la toma de decisiones (Avramidis et al., 2005, Chokshi, 1999, Sencer et al., 2013), y muy usado en la comprobación tecnológica para la planificación (Gulati et al., 2001).

Con el objeto de contar en nuestras investigaciones con una herramienta de validación de los procesos de optimización no lineales, se recurre a la estadística para extraer de ella, algunos conceptos como el Análisis de Residuales y la Regla Empírica 68-95-99. Adicionalmente, se incorpora al análisis la evaluación de curtosis para datos muestrales que siguen una distribución normal, que combinados con una herramienta de simulación permiten especificar un método estadístico para evaluar el grado de convergencia de una serie numérica generada por un algoritmo. En el presente trabajo, se describe una estrategia para validar resultados y convergencia obtenidos a partir de algoritmos de optimización, denominada Validación Empírica Residual, que compara estadísticamente los resultados obtenidos por un algoritmo con los obtenidos por simulación, y bajo ciertas premisas de variabilidad estadística se puede concluir si el algoritmo es o no convergente. Este procedimiento se describe en la sección 2. En la sección 3 se presenta un caso práctico de validación de convergencia de un algoritmo de optimización no lineal entera aplicado al problema de Call Center. Finalmente, se expone las conclusiones en la sección 4.

\section{VALIDACIÓN EMPÍRICA RESIDUAL CON SIMULACIÓN}

\subsection{Análisis de Residuales}

El Análisis de Residuales es la herramienta que se utiliza para evaluar la idoneidad de un modelo de regresión lineal frente a un conjunto de datos experimentales mediante la definición de residuos y la comprobación de supuestos estadísticos a través del análisis de los gráficos de residuales (Asuero et al., 1989, Cook et al., 1982, Topp et al., 2004).

Los principales los supuestos estadísticos respecto del modelo de regresión que se comprueban con el gráfico de residuales son (Black, 2010, Cook et al., 1982, Massart et al., 2003, Verran et al., 1984):

1. La generación de residuos debe tener un comportamiento aleatorio y ser independientes e idénticamente distribuidas.

2. Todos los residuales deben tener igual varianza. Esto quiere decir, que la varianza debe ser constante en todo el rango de concentración dinámica de los residuales. Esta propiedad se conoce como homocedasticidad.

3. Todos los residuos son variables aleatorias con distribución (aproximadamente) normal con media 0, por lo que la esperanza residual debe ser 0.

La verificación de las propiedades como la de ser independiente e idénticamente distribuida, conduce al cumplimiento de otros supuestos implícitos (Korner-Nievergelt et al., 2015).

Comprobada la independencia residual implica el cumplimiento de supuestos como: $\boldsymbol{a}$ ) los residuales no se correlaciona con ninguna otra variable incluida o no en el modelo; b) Los residuos no están agrupados (es decir, las medias muestrales de cualquier conjunto de residuos son todas iguales); $\boldsymbol{c}$ ) Los residuos no están auto correlacionados (es decir, no existe autocorrelación temporal o espacial).

Por otro lado, decir que los residuos son idénticamente distribuidos, significa que: $\boldsymbol{a}$ ) Todos los residuos provienen de la misma distribución. En el caso de una regresión lineal, se asume que todos vienen de una misma distribución normal; b) La varianza residual es homogénea, es decir, se cumple la propiedad de homocedasticidad; ) La media de los residuos es cero en todo el rango de los valores predictores. Cuando los predictores numéricos (covariables) están presentes, implica que la relación entre la variable independiente y dependiente puede describirse adecuadamente mediante una línea recta en el plano.

La diferencia entre los valores observados de la variable dependiente $(y)$ y los valores que predice el modelo (人) se llama residual (e) (Martin et al., 2017). También se los conoce como error. Es decir:

Residuos $(e)=$ Valores observados $(y)$ - Valores que predice el modelo $(\hat{y}) \Rightarrow e=y-\hat{y}$

Donde $\sum \mathrm{e}_{i}=0$ y $\bar{e}=0$ siempre que se cumpla los supuestos estadísticos. 
En un gráfico residual se muestran los residuos en el eje vertical y la variable independiente en el eje horizontal. Cuando los puntos en el gráfico se dispersan aleatoriamente alrededor del eje horizontal, se considera que el modelo de regresión lineal es apropiado para los datos. Una manera rápida de verificar el supuesto de normalidad es analizar la tendencia del gráfico. Si los residuos se trazan aproximadamente a lo largo o sobre de la línea recta de la tendencia, entonces se cumple el supuesto de normalidad (Myers et al., 2009), lo que implica de inmediato satisfacer los supuestos de implicancia mencionados anteriormente. Desde el punto de vista conceptual, el análisis de residuales conforma una herramienta sencilla que facilita la detección errores y la comprobación del grado de aproximación entre una ecuación empírica o teórica con resultados experimentales (Tomàs et al., 2006).

\subsection{Regla Empírica o Regla 68-95-99}

Usar una estimación central como la media o la mediana junto con una medida de variación (como la desviación estándar o el rango intercuartil) en una distribución muestral es una buena manera de describir los valores o el comportamiento de una población. En el caso de que el histograma de frecuencias relativa de los datos tenga la forma de campana (o tiene una aproximación a la distribución normal), la media poblacional y la desviación estándar son la combinación adecuada para estudiar variabilidad o dispersión, y una regla especial los vincula para obtener información bastante detallada sobre la población en general. Esta regla es la llamada regla empírica, también conocida como regla 68-95-99.7 (Rumsey, 2016).

La regla empírica es una regla general que se usa para indicar el porcentaje aproximado de valores muestrales que se encuentran dentro del intervalo dado por las desviaciones estándares respecto de la media muestral, cuando éstos se distribuyen normal (Black, 2010). La regla se aplica, generalmente, a una variable aleatoria que sigue una distribución normal, con media $\mu$ y desviación estándar $\sigma$. Una característica importante expresa que, si la distribución de los datos es más o menos simétrica, unimodal y sigue una ley normal o una aproximación a ella, entonces aproximadamente el $68,27 \%$ de los datos se concentran dentro del rango $\mu \pm \sigma$, el $95,45 \%$ dentro de $\mu \pm 2 \sigma$, y $99,73 \%$ dentro de $\mu \pm 3 \sigma$. Un punto importante a tener en cuenta, es que la regla empírica no se aplica a conjuntos de datos con distribuciones muy asimétricas.

\subsection{Simulación}

La simulación es realizada mediante un simulador de Call Center desarrollado a los efectos de la investigación, cuyo modelo estocástico ha sido debidamente comprobado en (Barberis et al., 2011).

La particularidad de la herramienta es que implementa un dispositivo que permite cumplir dinámicamente la política de asignación de turnos pre-establecida. Este mecanismo no previsto en software de simulación de Call Centers comerciales, habilita y deshabilita cierta cantidad de agentes según la planificación de turnos estipulados, a medida que transcurre la simulación de una jornada laboral. De esta manera, las simulaciones que se realizan son muy cercanas a la realidad operativa de los Call Centers, lo que posibilita formular conclusiones satisfactorias y precisas respecto de la realidad objetiva.

La estocasticidad de las simulaciones por computadora depende de la generación de números pseudo-aleatorios. En ejecuciones sucesivas de simulación, la generación algorítmica de valores pseudo-aleagoritos generan resultados que violan los supuestos estadísticos del análisis de residuales, es decir, se generan estimaciones correlacionados, que como variables aleatorias, no serían independientes ni idénticamente distribuidas (Law et al., 2000). Para garantizar el proceso estocástico y evitar la problemática generada por la pseudo-aleatoriedad se puede recurrir a dos alternativas para generar muestras que superen test estadísticos de aleatoriedad e independencia:

1) Realizar $n$ ejecuciones distintas de simulación y extraer de ellos un subconjunto de $m(m<n)$ resultados de la variable de estudio, preferentemente los de mayor variabilidad. Esto dará lugar a $m$ observaciones. $\mathrm{O}$ bien,

2) Realizar $n$ grupos de $m$ ejecuciones distintas de simulación, con $n$ semillas distintas para la generación de pseudo-aleatorios (cada grupo utiliza la misma semilla para las $m$ simulaciones). Se conforma una matriz de tamaño $n \times m$. Luego, se diseña una nueva muestra aleatoria promediando los valores de cada columna de la matriz, generándose así, $m$ valores observados de simulación, con características de ser independientes.

De cualquiera de las dos alternativas se obtienen $m$ observaciones independientes, idénticamente distribuidas, y aproximadamente normal, muy convenientes para la generación de residuos. 


\subsection{Proceso de Validación Empírica Residual}

Lo que se quiere saber es si el algoritmo de optimización ejecutado con diferentes puntos iniciales del espacio de decisión converge a un mismo valor, y si éste es próximo o no a la solución del problema en estudio. Las conclusiones se desarrollan a partir de las comparaciones estadísticas entre las salidas de un proceso de simulación y los resultados deterministas obtenidos por el algoritmo objetivo.

El procedimiento consiste en generar las observaciones a partir de los resultados del algoritmo que se obtienen de $m$ ejecuciones con $m$ puntos diferentes de partida del espacio de decisión. Los procesos de simulación se inician también con los $m$ puntos diferentes usados por el algoritmo. Si $A_{\mathrm{i}}$ es el valor observado que se obtiene como resultado del algoritmo ejecutado en el $i$-ésimo experimento, y $S_{\mathrm{i}}$ la estimación de la misma variable de estudio obtenido por simulación en un proceso $i$-ésimo, entonces los residuos $r_{\mathrm{i}}=A_{\mathrm{i}}-S_{\mathrm{i}}$ para $i=1 \ldots m$, conforman la variable aleatoria de residuales $R$. Así, si $A_{\mathrm{i}} \rightarrow E\left(S_{\mathrm{i}}\right)$ entonces $E(R) \rightarrow 0$. Luego de generar la muestra, se realiza el análisis de residuales para asegurar el cumplimiento de los supuestos estadísticos. El incumplimiento de algunos de los supuestos, implica aumentar los tiempos de simulación y cambiar la alternativa de selección de resultados del proceso simulado. Si volviera a no cumplir algunos de los supuestos estadísticos del análisis de residuales, entonces se rechaza la hipótesis de que el algoritmo converge a la solución del problema en estudio. En el caso de verificar todos los supuestos, se considera que el algoritmo tiene una tendencia a converger a un punto límite. El paso siguiente es analizar el grado de concentración de los residuos alrededor de la medida central. La estimación se obtiene a partir del indicador de Curtosis, que señala el grado de apuntamiento o achatamiento de la distribución muestral (Khurshid et al., 2007). En el análisis, se compara la forma de la curva de la distribución muestral con la distribución normal estándar. Así, si el indicador de curtosis es mayor a 0, se tendrá una distribución muestral Leptocúrtica; si es igual a 0 será mesocúrtica, (indica que se tiene de una distribución normal); en cambio, si el indicador es menor a 0 se tendrá una distribución Platicúrtica (Chissom, 1970, Pearson, 1905). Para una aplicación exitosa de la regla empírica, es deseable un indicador de curtosis mayor o igual a cero, lo que dará lugar no sólo a una validación exitosa de la convergencia del algoritmo, sino también a una convergencia con un grado de precisión aceptable.

Satisfecha la condición de curtosis, se estudiará los resultados derivados del Desvío Estándar $(\sigma)$ en sus tres valores: $\sigma, 2 \sigma$ y $3 \sigma$, según la regla empírica 68-95-99. Es deseable que el $100 \%$ de los resultados algorítmicos estén dentro de un rango de aproximación aceptable alrededor de la solución. Al tratarse de un estudio estadístico, se buscará que al menos un cierto porcentaje de los resultados algorítmicos tengan una precisión aceptable, lo que implica fijar una cota superior a $2 \sigma$ ó $3 \sigma$, que dependerá del tipo de problema al que se quiere dar solución. Así por ejemplo, para el caso de estudio que se describe en la sección 3.2, en el que se busca determinar la política de distribución óptima de turnos laborables para los operarios de un Call Center, que maximicen los niveles de servicios (NS), se requiere que $2 \sigma$ ó $3 \sigma$ sea menor a 1 - puesto que, el NS se cuantifica en el rango real $(0,1]$ - para asegurar que al menos el $95 \%$ de los resultados algorítmicos estén dentro del rango de precisión.

\section{APLICACIÓN DE LA TÉCNICA DE VALIDACIÓN ALGORÍTMICA}

\subsection{El Problema a Resolver}

En el ámbito de los Call Centers, no hay soluciones algorítmicas que hayan obtenidos resultados óptimos claramente; ni mucho menos, hay consenso en cuanto a la mejor estrategia de implementación. Es por ello, que en la investigación que se desarrolla se trata de diseñar alternativas algorítmicas que buscan optimizar las políticas de planificación y distribución de turnos, desde la perspectiva de la Optimización No Lineal Entera. Así, el problema a resolver es de la forma:

$$
\begin{array}{ll}
\text { máx } & f(x) \\
\text { s.a. } & A x \geq r ; \\
& B x=\Omega ; \quad x \geq 0 ; x \in \square^{n}
\end{array}
$$


Donde $f(x)$ es la función objetivo no lineal con dominio sobre un espacio de decisión discreto, que mide el desempeño del Call Center en término de nivel de servicio (NS). La función es no derivable, no convexa y no configura un problema cuadrático. Las restricciones del problema son lineales convexa y se componen de una matriz $A \in^{\prime \mathrm{m} \times \mathrm{n}}$, y vectores $B, r \in^{\prime \mathrm{m}}, \mathrm{y} \Omega \in^{\prime}$.

El algoritmo seleccionado para el experimento es descripto en (Barberis et al., 2019) y tiene la particularidad de ser simple en su diseño y obtener resultados a una velocidad aceptable. En el pseudo-código que se muestra en la tabla $1, \mathrm{~F}(x)$ es una función de penalización de la forma $\mathrm{F}(x)=f(x)+\mathrm{P}(\mathrm{R}(x))$ donde $f(x)$ es la función objetivo, $\mathrm{R}(x)$ constituye las restricciones de (1) y $\mathrm{P}(r)$ es la función que devuelve 0 si $x$ cumple las restricciones del problema, y un valor ponderado negativo en caso de que no las cumpla. También se requiere ajustar los valores de las componentes reales de $x^{(\mathrm{k}+1)}$ al vector de enteros más próximo. La tarea de conversión es llevada a cabo por la función Ajustar en Entero(v) de la línea 10. Se trata de un proceso complejo que busca el vector integral más próximo al vector real que mejor evalúa a $\mathrm{F}(x)$.

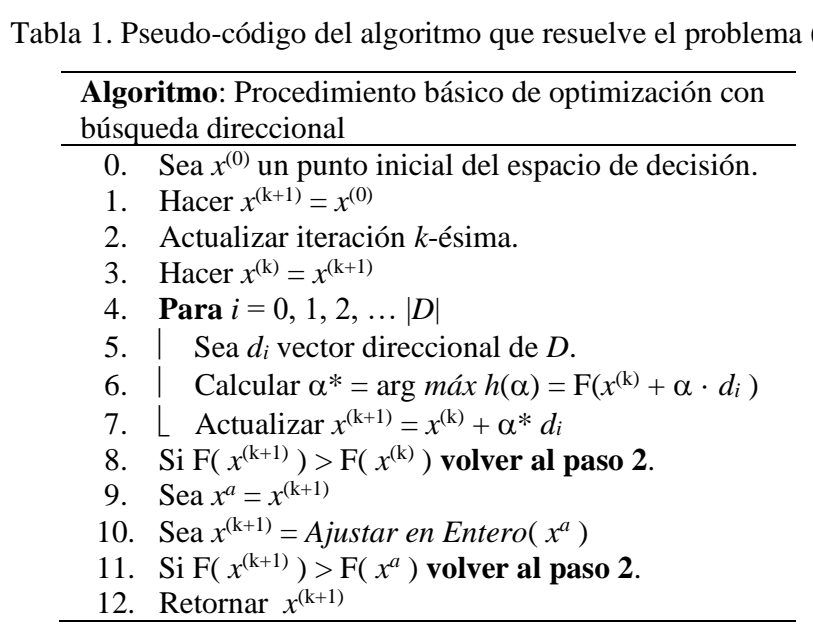

\subsection{Resultados Computacionales}

La validación del algoritmo, tiene por objetivo dar confiabilidad en la estabilidad y la convergencia hacia un resultado satisfactorio, considerando cierta precisión. Para tener mayor claridad en los experimentos, se muestra en detalle el análisis para un problema de dimensión 6 correspondiente a datos de un Call Center de pequeña envergadura, cuyos parámetros y solución son conocidos. Los parámetros utilizados son iguales tanto para el algoritmo como para el simulador, y se corresponde con: una jornada laboral de 9 hs.; turno de trabajo de 4 hs.; período de observación de 1 hora; AHT de 180 segundos; AWT de 20 segundos; media de abandono igual a 1.5; reintentos de llamadas del 60\%, y un nivel de servicio objetivo del $95 \%$.

En el experimento de simulación, se realizan 10 grupos de 20 réplicas (ejecuciones) independientes de una simulación de 5.000 jornadas. Se extraen de cada grupo 10 réplicas de mayor variabilidad y se promedian sus resultados. La tabla 2 muestra las 10 observaciones obtenidas como resultados del algoritmo y del experimento de simulación. La variable de estudio es NS y representa el valor de $f(x)$ en $(1)$ al ser evaluado en el punto del espacio de decisión. El vector de planificación inicial se representa en la tabla con la columna X, y representa el punto factible a partir del cual debe iniciarse la sucesión de aproximación. La comparación de la variable de estudio NS se realiza entre el modelo determinístico del algoritmo y el modelo estocástico de la simulación. Los resultados residuales se muestran en la última columna. 
Tabla 2. Residuos de 10 experimentos con puntos de partidas diferentes

\begin{tabular}{clccr}
\hline Experimento & X (de partida) & NS-Algoritmo & NS-Simulación & \multicolumn{1}{c}{ Residuos } \\
\hline 1 & $5,1,2,2,6,3$ & 0,988567835 & 0,988556579 & $1,1256 \mathrm{E}-05$ \\
2 & $5,2,1,2,6,3$ & 0,988595998 & 0,988556676 & $3,9322 \mathrm{E}-05$ \\
3 & $5,3,0,5,3,3$ & 0,988545779 & 0,988556877 & $-1,1097 \mathrm{E}-05$ \\
4 & $5,4,0,7,0,3$ & 0,988569574 & 0,988556239 & $1,3334 \mathrm{E}-05$ \\
5 & $5,5,3,2,1,3$ & 0,988555999 & 0,988557011 & $-1,0118 \mathrm{E}-06$ \\
6 & $5,6,2,0,3,3$ & 0,988556784 & 0,988555922 & $8,623 \mathrm{E}-07$ \\
7 & $5,7,2,0,2,3$ & 0,988549359 & 0,988556722 & $-7,3629 \mathrm{E}-06$ \\
8 & $5,8,1,0,2,3$ & 0,988565779 & 0,988556658 & $9,1206 \mathrm{E}-06$ \\
9 & $5,9,1,1,0,3$ & 0,988549496 & 0,988557104 & $-7,6083 \mathrm{E}-06$ \\
10 & $5,5,0,6,0,3$ & 0,988556779 & 0,988556848 & $-6,89 \mathrm{E}-08$ \\
\hline & & & $\sum \mathrm{e}=$ & $4,6746 \mathrm{E}-05$ \\
& & & $\bar{e}=$ & $4,6746 \mathrm{E}-06$ \\
& & & Durbin-Watson= & $1,404909 \mathrm{E}-05$ \\
& & & Curtosis $=$ & 0,41663062 \\
& & &
\end{tabular}

Suponiendo que los resultados dados por el algoritmo son comparables con los obtenidos por simulación, entonces el análisis de residuales debe mostrar que se cumplen los siguientes supuestos:

- Los residuales están centradas en torno al eje de abscisas (para verificar el supuesto de normalidad).

El supuesto de normalidad se verifica con el gráfico de la figura 1, que muestra la cercanía y la distribución de los residuos muestrales alrededor del eje de abscisa.

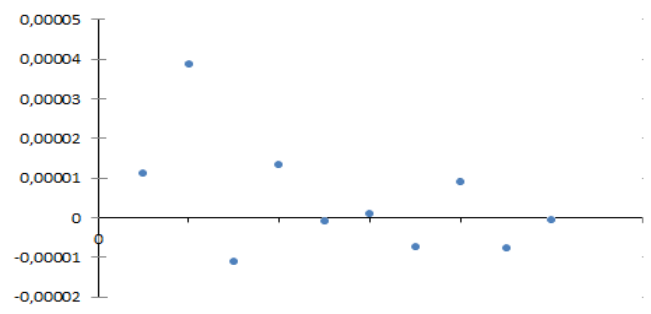

Figura 1. Gráfico de residuales

Del análisis gráfico, se deduce el cumplimiento del supuesto de normalidad.

- La distribución de los residuales sigue la ley normal de probabilidad (la sumatoria de los residuales y el promedio muestral deben tender a cero).

La tabla 2 muestra que $\sum \mathrm{e}_{i} \rightarrow 0$ y $\bar{e} \rightarrow 0$, y verificado el supuesto de normalidad con el gráfico de residuales, se acepta que la muestra tiene una distribución aproximadamente normal con media muestral tendiente a cero.

- Los residuales no presentan autocorrelación, es decir, la muestra se conforma con residuos independiente entre sí.

Para verificar que estadísticamente no hay auto-correlación (son independientes) se usará el indicador de Durbin-Watson (Durbin et al., 1950), cuya expresión se muestra en la figura 2, que toma valor 2 cuando los residuos son completamente independientes. Si $D W<2$ indica auto-correlación positiva, y $D W>2$ se trata de una autocorrelación negativa. En la práctica cuando $D W$ se encuentra entre 1.5 y 2.5 se considera que existe independencia. Para ello, se compara el estadístico $D W$ con valores críticos según el nivel de significancia $\alpha$. 
Los valores se encuentran tabulados para valores límites $d_{\mathrm{L}}$ y $d_{\mathrm{U}}$ (Durbin et al., 1951). Para determinar el criterio de decisión, se toma en cuenta el estadístico $D W$ calculado en la tabla 2. Dicho valor es $D W=2,21663062$. Para determinar los valores críticos $d_{\mathrm{L}}$ y $d_{\mathrm{U}}$ para un nivel de significancia del $5 \%$, son necesarios el número de muestras $n=10 \mathrm{y}$ la cantidad de variables independientes $k=1$.

Los valores críticos obtenidos de la tabla Durbin-Watson con 5\% de significancia son:

$$
d_{\mathrm{L}}=0,879 \Rightarrow 4-d_{\mathrm{L}}=3,121 \quad d_{\mathrm{U}}=1,320 \Rightarrow 4-d_{\mathrm{U}}=2,68
$$

Se contrasta la hipótesis nula $\mathrm{H}_{0}=$ Los residuos no están auto-correlacionados contra $\mathrm{H}_{a}=$ Los residuos están auto-correlacionados. Como $D W \in\left[d_{\mathrm{U}} ; 4-d_{\mathrm{U}}\right]=[1,320 ; 2,68]$ no se rechaza la hipótesis nula $\mathrm{H}_{0}$. Con lo cual, se verifica el supuesto de independencia entre los residuos muestrales.

\section{- La dispersión de los residuales es constante (homocedasticidad).}

Para demostrar la homocedasticidad se usa del Test de Bartlett (Bartlett, 1937). Éste define la hipótesis nula, $\mathrm{H}_{0}$ que las varianzas de $k$ muestras independientes de una población son iguales, frente a la hipótesis alternativa de que al menos dos son diferentes. El estadístico se muestra en la figura 3.

$$
D W=\frac{\sum_{i=2}^{n}\left(e_{i}-e_{i-1}\right)^{2}}{\sum_{i=1}^{n} e_{i}^{2}} ; \quad 0 \leq D W \leq 4
$$

Figura 2. Indicador de Durbin-Watson

$$
\begin{aligned}
& T=\frac{(N-k) \ln \left(S_{p}^{2}\right)-\sum_{i=1}^{k}\left(n_{i}-1\right) \ln \left(S_{i}^{2}\right)}{1+\frac{1}{3(k-1)}\left\{\left(\sum_{i=1}^{k} \frac{1}{n_{i}-1}\right)-\frac{1}{N-k}\right\}} \\
& \text { donde } N=\sum_{i=1}^{k} n_{i} \quad ; \quad S_{p}^{2}=\frac{\sum_{i=1}^{k}\left(n_{i}-1\right) S_{i}^{2}}{N-k}
\end{aligned}
$$

Figura 3. Estadístico de Bartlet

El estadístico de prueba $T$ tiene una distribución aproximadamente $\chi_{k-1}^{2}$. Por lo que, la hipótesis nula se rechaza si $T>\chi_{k-1, \alpha}^{2}$ con un grado de significancia $\alpha$.

Teniendo en cuenta los valores residuales de la tabla 2, se forman 6 grupos diferentes de 5 elementos residuales cada una, seleccionados al azar. Se calculan las varianzas respectivas de cada grupo y se los utiliza para calcular el estimador de Bartlett.

El estadístico de Bartlett que se estima es $T=6,8778$, y el valor crítico con significancia del $5 \%$ que es lo habitual es $\chi_{5 ; 0.05}^{2}=11,0705$. Dado que $T<\chi_{5 ; 0.05}^{2}$ entonces no se rechaza la $\mathrm{H}_{0}$, por lo que el supuesto de homocedasticidad queda verificado, y el supuesto de normalidad completamente comprobado.

De esta manera, se verifica el cumplimiento de todos los supuestos del Análisis de Residuales. Por lo que se puede concluir que el algoritmo converge estadísticamente a la solución del problema (1).

\section{- Análisis de Curtosis}

El paso siguiente consiste en analizar el grado de precisión de la convergencia. Para ello, se procede a medir el grado de apuntamiento o achatamiento de la distribución aproximadamente normal de la muestra de residuales, lo que se logra con el indicador de curtosis. La tabla 2 muestra el cálculo del indicador de curtosis según (DeCarlo, 1997), cuya expresión se muestra en la figura 4.

Al ser C >0 muestra que se trata de una distribución con curtosis positiva del tipo leptocúrtica que es la deseada. Por otro lado, algunos investigadores expresan que los momentos muestrales $m_{\mathrm{r}}$ no son estimaciones insesgadas de los momentos poblacionales $\mu_{\mathrm{r}}$, y proponen otra expresión para el indicador de curtosis que es insesgado para distribuciones normales (Joanes et al., 1998). La expresión se muestra en la figura 5, donde $S$ denota el desvío típico. Teniendo en cuenta la muestra de residuales de la tabla 2, el estimador de curtosis insesgado es $K=2,878598261$ que es ampliamente mayor a cero $(K>0)$. 


$$
C=\frac{\sum\left(X_{i}-\bar{X}\right)^{4} / n}{\left[\sum\left(X_{i}-\bar{X}\right)^{2} / n\right]^{2}}-3=0,445
$$

Figura 4. Curtosis según (DeCarlo, 1997)

$$
K=\left\{\frac{n(n+1)}{(n-1)(n-2)(n-3)} \sum\left(\frac{x_{j}-\bar{x}}{s}\right)^{4}\right\}-\frac{3(n-1)^{2}}{(n-2)(n-3)}=2,879
$$

Figura 5. Curtosis según (Joanes et al., 1998)

Ambos indicadores de curtosis prueban que la distribución muestral es del tipo leptocúrtica. Esto significa que la mayoría de las observaciones residuales se ubica en un entorno cercano a la esperanza $E(e)=0$.

Teniendo en cuenta el resultado anterior, y por aplicación de la regla empírica 68-95-99, se tiene que el 99,7\% de las observaciones se concentran en el rango, ya sea en $[\bar{x}-3 \sigma ; \bar{x}+3 \sigma]$ o bien en $[\mu-3 \sigma ; \mu+3 \sigma]$. Dado que NS - medido a través de $f(x)$ en el problema (1) - es un indicador porcentual que se miden en el rango $(0,1]$, y si $3 \sigma<1$, entonces la magnitud del error de aproximación del resultado del algoritmo es del mismo orden de magnitud del desvío estándar $(\sigma)$. En el experimento $\sigma=0,00001404909$, por lo que, aproximadamente, el resultado final del algoritmo tiene al menos 4 dígitos de precisión, que se considera muy satisfactorio.

\section{CONCLUSIONES}

El análisis de Residuales es un proceso estadístico que sirve para estudiar características de aproximación en modelos de regresión. En este trabajo, se muestra a dicho proceso como un recurso clave en el estudio de la convergencia de algoritmos de optimización. La combinación del indicador de curtosis con la regla empírica 68-95-99 posibilitó estimar el grado similitud entre los resultados obtenidos por el algoritmo y los de simulación. La técnica también fue aplicada a problemas más grandes, de hasta dimensión 1000, usando el mismo algoritmo mostrado en la sección 3.1, en el que es difícil conocer la solución global, por lo que se confía en las estimaciones obtenidas por el simulador. En todos los casos se han obtenido resultados de validación de convergencia muy satisfactorios, por lo que, la técnica de validación empírica de convergencia algorítmica proporciona un marco dentro del cual, diferentes algoritmos podrían compararse y contrastar su convergencia a un punto límite, tan cercano como se quiera de la solución del problema.

\section{REFERENCIAS}

Asuero, A. G. and Gonzalez, A. G. (1989) Some observations on fitting a straight line to data. Microchemical Journal, 40 (2), 216-225.

Atlason, J., et al. (2007) Optimizing Call Center Staffing Using Simulation and Analytic Center Cutting-Plane Methods. Management Science, 54 (2), 295-309.

Avramidis, A. N. and L' Ecuyer, P. (2005) Modeling and simulation of call centers. In: the Winter Simulation Conference. Orlando, FL, USA: IEEE, pp. 144-152.

Barberis, A. R. and Del Moral Sachetti, L. E. (2011) Modelización, Simulación y Optimización del personal operativo en la administración de Call/Contact Center. In: Simposio de Investigación Operativa 2011 - 40 JAIIO. Córdoba, Argentina: SADIO, pp. 103-120.

Barberis, A. R. and Del Moral Sachetti, L. E. (2019) Programación No Lineal Entera en la Planificación Óptima de Turnos para un Modelo de Call Center. In: VII Congreso de Matemática Aplicada, Computacional e Industrial MACI 2019. S10-Métodos Numéricos: Algoritmos y Aplicaciones. Río Cuarto, Córdoba. Argentina: ASAMACI, Vol. 7, pp. 201-204.

Bartlett, M. S. (1937) Properties of Sufficiency and Statistical Tests. Proceedings of the Royal Society of London. Series A, Mathematical and Physical Sciences, 160 (901), 268-282.

Black, K. (2010) Business Statistics: For Contemporary Decision Making. 6 ed. John Wiley \& Sons, Inc. USA.

Caprara, A., et al. (2003) Models and algorithms for a staff scheduling problem. Mathematical Programming, 98(1), 445-476.

Chissom, B. S. (1970) Interpretation of the Kurtosis Statistic. The American Statistician, 24 (4), 19-22. 
Chokshi, R. (1999) Decision support for call center management using simulation. In: Proceedings of the 31st conference on Winter simulation: Simulation---a bridge to the future - Volume 2. Phoenix, Arizona, USA: Association for Computing Machinery, Vol. 2, pp. 1634-1639.

Cook, R. D. and Weisberg, S. (1982) Residuals and Influence in Regression. (Monographs on Statistics and Applied Probability). University of Minnesota. School of Statistics. New York: Chapman and Hall.

DeCarlo, L. T. (1997) On the meaning and use of kurtosis. Psychological Methods, 2 (3), 292-307.

Durbin, J. and Watson, G. S. (1950) Testing for Serial Correlation in Least Squares Regression: I. Biometrika, 37 (3/4), 409-428.

Durbin, J. and Watson, G. S. (1951) Testing for Serial Correlation in Least Squares Regression. II. Biometrika, 38 (1/2), 159-177.

Gulati, S. and Malcolm, S. A. (2001) Call center scheduling technology evaluation using simulation. In: the Winter Simulation Conference. Arlington, VA, USA: IEEE, Vol. 2, pp. 1438-1442.

Ingolfsson, A., et al. (2002) Accounting for time-varying queueing effects in workforce scheduling. European Journal of Operational Research, 139 (3), 585-597.

Ingolfsson, A., et al. (2010) Combining integer programming and the randomization method to schedule employees. European Journal of Operational Research, 202 (1), 153-163.

Joanes, D. N. and Gill, C. A. (1998) Comparing measures of sample skewness and kurtosis. Journal of the Royal Statistical Society: Series D (The Statistician), 47 (1), 183-189.

Khurshid, A., et al. (2007) A note on finding peakedness in bivariate normal distribution using Mathematica. Pakistan Journal of Statistics and Operation Research, 3 (2), 75-86.

Kim, S.-M., et al. (2011) The Staffing Problem at the Call Center by Optimization and Simulation. IE Interfaces, 24 (1), 40-50.

Koole, G. and van der Sluis, E. (2003) Optimal Shift Scheduling with a Global Service Level Constraint. IIE Transactions, 35 (11), 1049-1055.

Korner-Nievergelt, F., et al. (2015) Chapter 6 - Assessing Model Assumptions: Residual Analysis. In: KORNER-NIEVERGELT, F., et al. (Eds.) Bayesian Data Analysis in Ecology Using Linear Models with R, BUGS, and STAN. Boston: Academic Press, pp. 75-94.

Law, A. M. and Kelton, W. D. (2000) Simulation Modelling and Analysis. (Series in Industrial Engineering and Management Science). 3 ed. McGraw-Hill Higher Education. New York, USA.

Martin, J., et al. (2017) Fitting Models to Data: Residual Analysis, a Primer - Chapter 7. In: HESSLING, J. P. (Ed.) Uncertainty Quantification and Model Calibration. Rijeka: IntechOpen, pp. 133-173.

Massart, D. L., et al. (2003) Chemometrics: a Textbook. (Data Handling in Science and Technology). Vol. 2. Elsevier Science B.V. Hungary.

Myers, R. H., et al. (2009) Response Surface Methodology: Process and Product Optimization Using Designed Experiments. (Wiley Series in Probability and Statistics). 3 ed. John Wiley \& Sons, Inc. Hoboken, New Jersey, USA.

Pearson, K. (1905) Das Fehlergesetz und Seine Verallgemeinerungen Durch Fechner und Pearson. A Rejoinder (Skew Variation, a Rejoinde). Biometrika, 4 (1/2), 169-212.

Robbins, T. R. and Harrison, T. P. (2010) A stochastic programming model for scheduling call centers with global Service Level Agreements. European Journal of Operational Research, 207 (3), 1608-1619.

Rumsey, D. J. (2016) Statistics For Dummies. 2 ed. Wiley Publishing, Inc. Indianapolis, Indiana, USA.

Sencer, A. and Basarir Ozel, B. (2013) A simulation-based decision support system for workforce management in call centers. SIMULATION, 89 (4), 481-497.

Tomàs, X., et al. (2006) Introducción al Cálculo Numérico. Institut Químic de Sarrià (IQS). Departament d'Estadística Aplicada. Barcelona, España.

Topp, R. and Gómez, G. (2004) Residual analysis in linear regression models with an interval-censored covariate. Statistics in medicine, 23 (21), 3377-3391.

Verran, J. A. and Ferketich, S. L. (1984) Residual Analysis for Statistical Assumptions of Regression Equations. Western Journal of Nursing Research, 6 (1), 27-40.

Zeltyn, S. and Mandelbaum, A. (2005) Call Centers with Impatient Customers: Many-Server Asymptotics of the M/M/n + G Queue. Queueing Systems, 51 (3), 361-402. 